\title{
Analysis of the relationship between Oxford classification, IgM deposition and multiple indexes and the adverse prognosis of patients with primary IgA nephropathy and related risk factors
}

\author{
YAN HAO, YULIANG ZHAO, RONGSHUANG HUANG and PING FU \\ Division of Nephrology, Kidney Research Laboratory, West China Hospital of \\ Sichuan University, Chengdu, Sichuan 610041, P.R. China
}

Received April 13, 2018; Accepted October 11, 2018

DOI: $10.3892 /$ etm.2018.7050

\begin{abstract}
The risk and influencing factors of prognosis in patients with primary $\operatorname{Ig}$ A nephropathy $(\operatorname{Ig} A N)$ were explored. One hundred and twenty-four patients who were diagnosed with IgA nephropathy in West China Hospital of Sichuan University were selected as the study subjects. The baseline data were recorded. All patients were followed up for 3 years. Patients with poor prognosis were defined as poor prognosis group, and the patient with no adverse prognosis was defined as a good prognosis group during the follow-up period. The risk factors that may affect the prognosis of patients with IgAN were analyzed by single factor analysis. The influence of all factors that were statistically significant on the prognosis of the patients was further evaluated by multifactor Cox regression. The single factor analysis and multivariate Cox proportional hazard model showed that patients with $24 \mathrm{~h}$ urinary protein, pathological type, Oxford classification (T1+T2), Lee (grade IV) and mesangial IgM deposition were independent factors of patients, and the difference was statistically significant, their P-values were 0.041, 0.046, 0.037, 0.043 , and 0.028 , respectively. Patients with $24 \mathrm{~h}$ urinary protein, pathological type, Oxford classification (T1+T2), Lee (grade IV) and mesangial IgM deposition can be used as independent factors affecting poor prognosis in primary IgAN patients. It provides evidence for early detection of high-risk IgA nephropathy.
\end{abstract}

\section{Introduction}

$\operatorname{Ig}$ A nephropathy ( $\operatorname{Ig} \mathrm{AN})$ is the most common primary glomerular disease in China. Its incidence rate is increasing

Correspondence to: Dr Ping Fu, Division of Nephrology, Kidney Research Laboratory, West China Hospital of Sichuan University, 37 Guoxue Lane, Wuhou, Chengdu, Sichuan 610041, P.R. China E-mail: f7r2ww@163.com

Key words: IgA nephropathy, IgM deposition, Oxford classification year by year. The disease condition of many patients is chronically progressive. Approximately $15-40 \%$ of patients will progress to end-stage renal disease after 10 years. Kidney replacement therapy is needed to maintain their lives, which brings great burden to patients and society (1). Although process of IgAN development is relatively benign, it progressively developes and has a poor prognosis, which lead to the fact that it becomes the main cause of chronic renal failure (2). Therefore, it is one of the key steps to improve the level of treatment as finding indicators related to disease activity, progression and prognosis. It also has important guiding significance for clinical work.

According to previous studies, many factors are closely related to the prognosis of $\operatorname{Ig} \mathrm{AN}$, such as severity of illness, $24 \mathrm{~h}$ urinary protein, GFR levels, and creatinine clearance (Ccr). These factors also have potential clinical predictive value. With the deepening and widening of research, more and more factors have been confirmed to be associated with IgAN. The influence of pathological type and family history on the outcome of disease treatment and prognosis are reflected in the research of Kusano et al (3). It was found by Yabuki et al (4) that $\operatorname{IgAN}$ patients with $\operatorname{IgM}$ deposition had higher urinary protein compared with those without IgM deposition. Some factors may affect the prognosis of patients with $\operatorname{Ig} \mathrm{AN}$ to some extent, such as more immunofluorescence deposition, more severe pathological lesions and $\operatorname{IgM}$ deposition. These factors affecting IgAN are numerous and complex. The research on the analysis of the disease prognosis and its impact of the overall factors is not common in China. Therefore, the authors wanted to compare the general data and clinical data of $\operatorname{IgAN}$ patients with good prognosis and poor prognosis, analyze the value of Oxford classification, IgM deposition, and multiple indicators that may affect the prognosis of patients in predicting the prognosis risk of $\operatorname{IgAN}$ patients. The purpose is to provide the basis for the wide use of technology in pathological tests on $\operatorname{IgM}$ in clinical examination for IgAN patients.

\section{Patients and methods}

Enrolment. A total of 124 patients diagnosed with IgAN in West China Hospital of Sichuan University (Chengdu, China) 
from May 2012 to May 2014 were selected as study subjects. They included 55 males and 69 females. The age of patients was between 27 and 41 years. The average age was 33.96 years. This study was approved by the Ethics Committee of West China Hospital of Sichuan University (Chengdu, China). Signed informed consents were obtained from the patients or the guardians.

Inclusion/exclusion criteria. Inclusion criteria were: i) The patient was diagnosed with $\operatorname{IgAN}$ in the nephrology department according to the guideline criteria, and the number of glomeruli in the biopsy tissue was $>10$; and ii) the patient had no history of kidney transplant surgery. Exclusion criteria were: i) Secondary $\operatorname{IgAN}$, such as cirrhosis-associated nephritis, hepatitis B associated nephritis and anaphylactoid purpura nephropathy; ii) patients with acute heart failure, cardiogenic shock and other important organ diseases; and iii) clinical data was not complete.

Pathology detection method of kidney. All patients included in the study underwent ultrasound-guided percutaneous renal biopsy. Then the kidney tissues were obtained before treatment after diagnosis. Renal biopsy was embedded in paraffin. Serial sections in the thickness of 2-3 $\mu \mathrm{m}$ were stained through conventional hematoxylin and eosin (H\&E), periodic acid iodine, periodic acid-silver metheramine staining and Masson staining. Immunohistochemistry was used to detect the expression intensity and deposition site of IgG and IgM by direct immunofluorescence. The sections were washed twice with PBS for 3 min each time, dripped with rabbit anti-human IgG and rabbit anti-human IgM monoclonal antibodies (1:100; cat nos. ab193172 and ab212201; Abcam, Cambridge, MA, USA), incubated in water bath at $37^{\circ} \mathrm{C}$ for $60 \mathrm{~min}$, washed twice with PBS for 3 min each time. Then incubate with Alexa Fluor ${ }^{\circledR}$ 488 goat anti-rabbit secondary polyclonal antibody (1:200; Cat nos.ab150077; Abcam, Cambridge, MA, USA). Then the samples were observed under fluorescence microscope after sealed with glycerol. Pathological data included data of endothelial cell proliferation, segmental sclerosis, nevus necrosis, mesangial proliferation, proportion of sclerosing, percentage of crescents, and extent of tubulointerstitial injury. Kidney pathology was performed by Lee and Oxford classification. Katafuchi semi-quantitative scoring method was used to score the glomerular, renal tubulointerstitial, and renal angiopathy. All the analysis of sections were performed independently on the premise of unknown clinical outcome of patients. When there were inconsistencies or doubts among pathologists and clinical investigators, these were submitted to a higher-level pathologist for review. The Lee classification included 5 grades. Grade I: Glomerular function was almost normal with occasional mesangial mild hyperplasia, grade II: Glomerular mesangial widening accompanied by cell hyperplasia (more than 50\%) without small crescents in general, grade III: Mesangial diffuse widening with cell proliferation, interstitial edema and cell infiltration, grade IV: Patients with diffuse mesangial hyperplasia and sclerosis, and glomerular appearance (more than 50\%), with tubular atrophy and interstitial inflammation, and grade V: Symptoms was similar to IV but more severe. Oxford classification: Ml for mesangial cell proliferation $>0.5$; E1 for endothelial cell proliferation; s1 for segmental sclerosis; T1 for renal tubular atrophy; and T2 for interstitial fibrosis $>25 \%$.

Treatment. According to the Kidney Disease: Improving Global Outcomes (KDIGO) IgA nephropathy treatment guidelines, treatments are as follows: i) ARB or ACEI: ARB or ACEI is the preferred medicine for patients with proteinuria or hypertension. ii) Glucocorticoid indications: a) After 3-6 months of treatment with ARB or ACEI, urinary protein in $24 \mathrm{~h}$ was still above $1 \mathrm{~g}$ and glomerular filtration rate was above $50 \mathrm{ml} / \mathrm{min}$; b) patients with clinical manifestations of nephrotic syndrome and pathological manifestations of minimal change nephropathy; c) crescentic IgA nephropathy; and d) patients with rapid decline in renal function. iii) The application of immunosuppressive agents: crescentic IgA nephropathy with rapid decline in renal function may consider glucocorticoid combined with cyclophosphamide treatment, no other use of immunosuppressive agents is recommended. iv) Others: a) Fish oil: Although the efficacy of fish oil therapy in patients with IgA nephropathy is inaccurate, it is of low risk and has cardiovascular benefits. Therefore, fish oil treatment is still considered safe; b) antiplatelet drugs: Not recommended for use; and c) tonsillectomy: The curative effect is inaccurate and further large sample evaluation is needed.

Data collection. This study prospectively analyzed patients and the data included baseline data such as $24 \mathrm{~h}$ urinary protein, GFR levels, endogenous creatinine clearance (Ccr), erythropoietin (EPO), and urinary $\mathrm{N}$-acetyl- $\beta$-D-glucosidase (NAG). Baseline data included age on onset, sex, family history (IgAN in close relatives of the third generation of the family), and pathological type. The method of GFR measurement is complex. Intravenous injection of $24 \%$ inulin or isotopic markers secreted by the renal tubules is required. GFR is often estimated by various equations in clinical practice. The information of Scr, age, and sex are needed for estimation. Among them, one of the best is the Ruijin equation used to estimate GFR: $234.96 \times$ Scr $-0.926 \times$ age $-0.280 \times$ a (a, female $=0.828$, male $=1)$. Cor is also estimated by using the Cockcroft-Gault formula: $\mathrm{Ccr}=(140$-age $)$ x body weight $(\mathrm{kg}) /[72 \mathrm{x} \mathrm{Scr}(\mathrm{mg} /$ $\mathrm{dl})]$ or $\mathrm{Ccr}=[(140$-age $) \times$ body weight $(\mathrm{kg})] /[0.818 \times \mathrm{Scr}$ $(\mu \mathrm{mol} / \mathrm{l})]$, females are calculated as $\mathrm{x} 0.85$.

Follow-up. All the 124 patients involved in the study were followed up for 3 years. The first follow-up was 1 month after the end of treatment, followed by a telephone interview every 3 months. The end point event was: The patient had a poor prognosis from the time of enrollment during the follow-up period. The poor prognosis included: i) GFR decreased by more than 50\% compared to baseline values. ii) Patients were admitted to end-stage renal disease (ESRD) or required continuity of the kidney replacement therapy or death from kidney disease. The final follow-up records were used as follow-up results to record the number of patients who had a poor prognosis. Censorship was defined as patients of the study who were lost to follow-up, access denied, withdrawal midway, and dying from other reasons unrelated to the study.

Follow-up grouping and data arrangement. Patients with poor prognosis during the follow-up period were defined as poor 
Table I. Clinical data, detection indicators and pathological features of 124 patients.

\begin{tabular}{lc}
\hline Index & $($ mean $\pm \mathrm{SD}) / \mathrm{n}$ \\
\hline Age (year) & $33.63 \pm 7.63$ \\
Course of disease (month) & $7.72 \pm 3.97$ \\
Male (case) & 83 \\
Female (case) & 41 \\
Family history (case) & 34 \\
Systolic blood pressure (mmHg) & $139.29 \pm 40.12$ \\
Diastolic blood pressure (mmHg) & $87.93 \pm 29.02$ \\
GFR (ml/min) & $92.53 \pm 33.64$ \\
Ccr (ml/min) & $106.39 \pm 41.92$ \\
EPO (IU) & $36.36 \pm 11.53$ \\
NAG (U/l) & $26.73 \pm 8.64$ \\
IgG deposition (case) & 19 \\
IgM deposition (case) & 27 \\
24 h urine protein quantity & \\
$<1.0$ g (case) & 84 \\
$1.0-3.5$ (case) & 27 \\
$>3.5$ (case) & 13 \\
\hline
\end{tabular}

Index

Pathological type

Mesangial proliferative glomerulonephritis (case)

Endocapillary proliferative glomerulonephritis (case)

Membranoproliferative nephritis (case)

Crescentic glomerulonephritis (case)

Proliferative sclerotic nephritis (case)

Lee grades

I (case)

II (case)

III (case)

IV (case)

$\mathrm{V}$ (case)

41

12

8

Oxford classification

M1 (case)

E1 (case)

S1 (case)

$\mathrm{T} 1+\mathrm{T} 2$ (case)

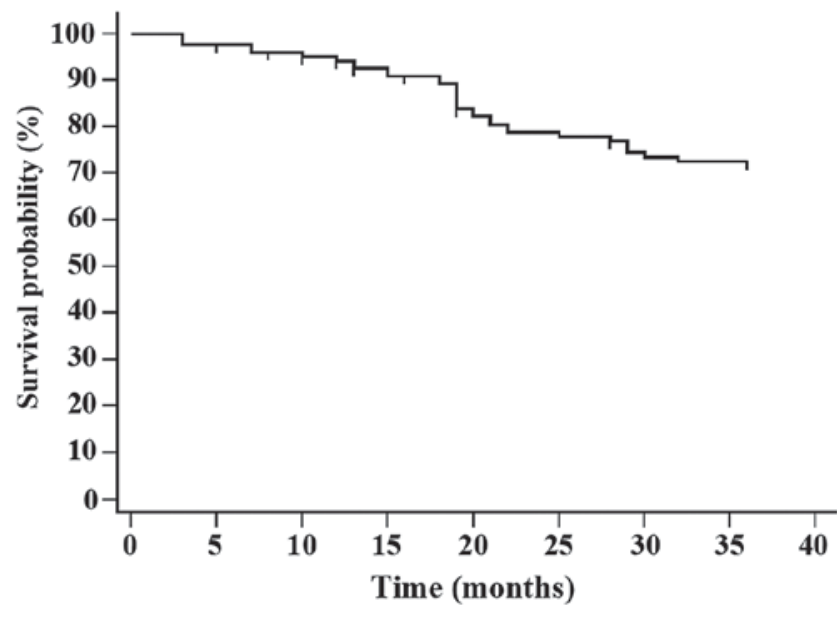

Figure 1. Survival curve analysis of poor prognosis in patients with IgAN.

the poor prognosis rate and the survival curve was drawn. Univariate analysis was used to explain the factors which may have an impact on the prognosis of IgAN patients. At the same time, a multi-factor Cox proportional hazards model was established to further analyze the factors that may have a significant impact to explore the impact of each variable on the risk of IgAN patients.

\section{Results}

Basic data analysis. In this study, 124 subjects were included. The clinical data, detection indicators and pathological features of all patients are shown in Table I.

Analysis of survival curve of patients with poor prognosis. At the end of follow-up, the results showed that within the total 124 patients, there were 10 patients lost to follow-up, 82 patients with good prognosis (defined as good prognosis), 32 patients with poor prognosis (defined as poor prognosis), and 19 of them had a decrease in GFR more than $50 \%, 9$ people entered end-stage renal disease, and 4 died. Kaplan-Meier survival curves suggest that the rate of poor prognosis of $\operatorname{IgAN}$ patients is $28.07 \%$ (Fig. 1).

Univariate analysis offactors that may influence the prognosis of patients with IgAN. Factors that may affect the prognosis of the disease were individually included in the Cox proportional hazards model for univariate analysis. The results showed that within the baseline data, family history, pathological type, and Lee classification (IV and V) affected the prognosis of the disease. Statistical significance was set at $\mathrm{P}<0.05$. Among other factors, urinary protein in $24 \mathrm{~h}$, GFR level, Ccr, IgM deposition in the mesangial area and Oxford classification (T1+T2) could affect the prognosis of IgAN patients. The difference was statistically significant at $\mathrm{P}<0.05$ (Table II).

Multivariate Cox proportional regression analysis of factors affecting prognosis of IgAN patients. With further analysis of the above results, multivariate Cox regression results showed that urinary protein in $24 \mathrm{~h}$, pathological type, Oxford classification (T1+T2), Lee grade (grade IV) and IgM deposition
Statistical analysis. SPSS 19.0 (SPSS, Inc., Chicago, IL, USA) scoring statistical software was used for analysis. Survival analysis was performed by Kaplan-Meier method to calculate prognostic groups. Patients without worsening were defined as having a good prognosis. At the end of follow-up, the clinical data such as baseline data, $24 \mathrm{~h}$ urinary protein, GFR level, Ccr, EPO, urinary NAG, IgG and IgM deposition in the mesangial area and Oxford classification were analyzed. 
Table II. Single factor Cox regression analysis on the prognosis of patients.

\begin{tabular}{|c|c|c|c|c|c|c|c|c|}
\hline \multirow[b]{2}{*}{ Characteristics } & \multirow[b]{2}{*}{ B } & \multirow[b]{2}{*}{ SE } & \multirow[b]{2}{*}{ Wald } & \multirow[b]{2}{*}{ df } & \multirow[b]{2}{*}{ P-value } & \multirow[b]{2}{*}{ RR } & \multicolumn{2}{|c|}{$95.0 \% \mathrm{CI}$} \\
\hline & & & & & & & Lower limit & Upper limit \\
\hline Age & 0.011 & 0.173 & 4.385 & 1 & 0.594 & 1.011 & 0.720 & 1.419 \\
\hline Sex & -0.409 & 0.325 & 2.113 & 1 & 0.783 & 0.664 & 0.351 & 1.255 \\
\hline Family history & 0.218 & 0.102 & 2.084 & 1 & 0.041 & 1.244 & 1.019 & 1.519 \\
\hline Pathological type & 0.443 & 0.174 & 2.842 & 1 & 0.033 & 1.642 & 0.456 & 0.903 \\
\hline \multicolumn{9}{|l|}{ Lee grades } \\
\hline $\mathrm{I}$ & & & & & 0.279 & 1 & & \\
\hline II & -0.282 & 0.273 & 3.753 & 1 & 0.174 & 0.754 & 0.442 & 1.288 \\
\hline III & 0.158 & 0.375 & 4.653 & 1 & 0.062 & 0.854 & 0.409 & 1.781 \\
\hline IV & 0.261 & 0.123 & 4.975 & 1 & 0.028 & 1.298 & 1.020 & 1.652 \\
\hline V & 0.233 & 0.107 & 4.128 & 1 & 0.037 & 1.262 & 1.023 & 1.566 \\
\hline $24 \mathrm{~h}$ urinary protein & 0.351 & 0.106 & 5.424 & 1 & 0.031 & 1.421 & 1.154 & 1.749 \\
\hline GFR & 0.583 & 0.118 & 9.389 & 1 & 0.002 & 1.792 & 1.422 & 2.258 \\
\hline $\mathrm{Ccr}$ & 0.436 & 0.147 & 9.689 & 1 & 0.001 & 1.546 & 1.159 & 2.062 \\
\hline EPO & -0.167 & 0.232 & 2.374 & 1 & 0.397 & 0.846 & 0.537 & 1.333 \\
\hline NAG & -0.078 & 0.218 & 3.965 & 1 & 0.137 & 0.925 & 0.603 & 1.418 \\
\hline $\mathrm{IgG}$ & 0.128 & 0.216 & 2.894 & 1 & 0.157 & 0.745 & 1.736 & 2.145 \\
\hline $\operatorname{IgM}$ & 0.513 & 0.187 & 3.71 & 1 & 0.034 & 1.670 & 1.158 & 2.409 \\
\hline \multicolumn{9}{|l|}{ Oxford classification } \\
\hline M1 & & & & & 0.362 & 1 & & \\
\hline E1 & -0.178 & 0.183 & 3.846 & 1 & 0.263 & 0.837 & 0.585 & 1.198 \\
\hline $\mathrm{S} 1$ & -0.089 & 0.238 & 5.745 & 1 & 0.121 & 0.915 & 0.574 & 1.459 \\
\hline T1-T2 & 0.291 & 0.125 & 3.175 & 1 & 0.012 & 1.338 & 1.047 & 1.709 \\
\hline
\end{tabular}

Ccr, creatinine clearance; EPO, erythropoietin; NAG, N-acetyl- $\beta$-D-glucosidase.

in the mesangial area are independent factors influencing patients. The difference was statistically significant (Table III). The P-values were 0.041, 0.046, 0.037, 0.043, and 0.028, respectively (data not shown).

\section{Discussion}

At the time $\operatorname{Ig} \mathrm{AN}$ was reported, $\mathrm{Ig} \mathrm{AN}$ was considered to be a glomerular disease with a good prognosis. Most of the patients were able to achieve a good response after receiving treatment (5). With the deepening of research, IgAN has gradually developed and individual differences are relatively large. Although there are some good clinical treatments, some patients will still enter end-stage renal disease after treatment. In this study, Kaplan-Meier survival curves were drawn for the survival of patients with $\operatorname{IgAN}$. The results showed that in 124 subjects, the survival rate of IgAN kidneys was $94 \%$ at 10 months, $89 \%$ at 20 months, and $78 \%$ at 25 months and $72 \%$ at 30 months. It suggested that $\mathrm{Ig} \mathrm{AN}$ patients showed progressive development and the rate of progression to end-stage renal failure was fast, which was consistent with the results of long-term follow-up studies of IgAN patients by scholar Ohata et al (6).

There are many indicators of prognostic risk and related risk factors in IgAN patients. Traditional indicators include age, sex, family history, Scr and GFR. In the study of D'Amico (7), GFR was considered to be the most powerful laboratory indicator of IgAN prognosis. The results of this study also showed that GFR had an impact on disease prognosis. The calculation of GFR involves multiple indicators such as age, sex, and Scr, and the assessment of IgAN's progress is more accurate (8). This report is slightly different focusing on the factors affecting the prognosis of IgAN patients and improvements which can prevent patients from having a poor prognosis. The severity of the patient's condition has a great deal of clinical concern. From the point of pathological view, the patient's pathological changes are different, and each pathological type is of great significance in guiding the treatment.

For patients with medullary proliferative lesions as mainly pathological changes have visible hematuria, taking rhubarb mixture can inhibit the proliferation of mesangial cells. Patients with changes in glomerulosclerosis and interstitial fibrosis have large clinical manifestations of proteinuria, they can use either ARB (ACEI) or glucocorticoids according to specific needs (9). Patients with mesangial hyperplasia and glomerulosclerosis usually have interstitial lesions of different severity with different clinical manifestations, and the treatment methods may be determined based on the condition (10). In the view of the above-mentioned pathological types, the IgAN grading system has not yet been applied in 
Table III. Multiple factor Cox regression analysis on the prognosis of patients.

$95.0 \% \mathrm{CI}$

\begin{tabular}{|c|c|c|c|c|c|c|c|c|}
\hline \multirow[b]{2}{*}{ Factors } & \multirow[b]{2}{*}{ B } & \multirow[b]{2}{*}{ SE } & \multirow[b]{2}{*}{ Wald } & \multirow[b]{2}{*}{ df } & \multirow[b]{2}{*}{ P-value } & \multirow[b]{2}{*}{$\mathrm{RR}$} & \\
\hline & & & & & & & Lower limit & Upper limit \\
\hline Family history & 0.061 & 0.314 & 3.312 & 1 & 0.772 & 1.063 & 0.574 & 1.967 \\
\hline Pathological type & 1.150 & 0.575 & 3.995 & 1 & 0.046 & 3.159 & 1.023 & 6.758 \\
\hline Lee IV grades & & & & & 0.043 & 1 & & \\
\hline Lee $V$ grades & 0.021 & 0.321 & 3.721 & 1 & 0.169 & 1.021 & 0.544 & 1.915 \\
\hline GFR & 0.066 & 0.384 & 2.267 & 1 & 0.821 & 1.068 & 0.503 & 2.267 \\
\hline Ccr & 0.160 & 0.154 & 2.985 & 1 & 0.395 & 1.173 & 0.867 & 1.586 \\
\hline $24 \mathrm{~h}$ urinary protein & 0.345 & 0.081 & 2.743 & 1 & 0.041 & 1.412 & 1.205 & 1.655 \\
\hline $\operatorname{IgM}$ & 0.514 & 0.249 & 3.116 & 1 & 0.028 & 1.672 & 1.026 & 2.724 \\
\hline $\begin{array}{l}\text { Oxford classification } \\
(\mathrm{T} 1+\mathrm{T} 2)\end{array}$ & 0.264 & 0.113 & 7.338 & 1 & 0.037 & 1.302 & 1.043 & 1.625 \\
\hline
\end{tabular}

Ccr, creatinine clearance.

the world. The WHO histological classification method, the Hass classification method, the Lee classification method, and the Oxford classification score are commonly used. The first two are more concerned with diffuse lesions and sclerosis, while less attention is paid to crescents, renal tubules, and renal interstitial lesions. In the latter two methods, Lee classifications is more common and its objective is to consider various pathological changes such as glomerulosclerosis and interstitial lesions. The Oxford classification score is more predictive of value and is involved in renal tubules, glomeruli, and renal interstitium.

In this study, the joint application of the two-grading system is more comprehensive in describing the severity of the patient's condition. Previous studies have reported renal tubular and interstitial injury are the two independent risk factors that have the greatest adverse renal outcome (11), suggesting that Lee classification method and Oxford classification score have potential clinical significance in the prognosis of patients and can be used as an independent factor influencing the prognosis of patients. However, it still needs further analysis. Age affects the occurrence and development of many diseases, but specificity is not ideal generally. At present, its relationship with the prognosis of IgAN is still controversial. D'Amico conducted a comparative study of a certain number of IgAN patients and found that the age may have larger influence on the univariate analysis, which may affect the prognosis of patients. However, the conclusion of the single factor analysis in this study does not have the effect of age. After comparing the subjects included in this study with those of Sonoda et al (12) and Al Hussain et al (1), we found that the age of the subjects included in this study tends to be younger and more diffusely distributed. While the latter study suggests that age above 65 years is a risk factor for IgAN prognosis. It is speculated that the reason why the disease course and the prognosis of the disease are not independently related to each other may be the age distribution. The selection of sample size must also be considered. The selection of diastolic pressure, systolic blood pressure, EPO and NAG were all based on relatively recent literature reports. However, this study did not find statistical differences between the above factors in univariate analysis. The reason may be that most patients have taken ACEI or ARB. These drugs have a good effect on stabilizing blood pressure in patients (12).

In order to further explore the relationship between statistically significant factors and patient prognosis in the above single factor analysis. This study also used methods of multivariate Cox regression analysis. The analysis results suggested that the patient's $24 \mathrm{~h}$ urine protein, pathological type, Oxford classification (T1+T2), Lee classification (grade IV) and IgM deposition in mesangial area are independent factors of patients. Previous studies have suggested that deposition of IgM in the mesangial area is only non-specific immunodeposition and is an immunopathological response of the body (13). There is no pathological value and pathogenicity in the clinic. Subsequent studies have reported that $\operatorname{IgM}$ deposition in the mesangial area is a sign of worsening of the disease. Some scholars have also proposed that reducing the $\operatorname{IgM}$ antibody can delay the deterioration of the disease (14). At the same time, some scholars have also suggested that $\operatorname{IgM}$ deposition is related to $\mathrm{T}$ cell balance, production of multiple cytokines and activation of complement. In IgA nephropathy with IgM deposition, glomerular and tubulointerstitial lesions are often combined at the same time, resulting in an impact on prognosis (15), which is also consistent with the results of this study.

There are still deficiencies in this study. The number of subjects is small and the time of follow-up is short. There are not enough factors that may affect the prognosis of IgAN. In the follow-up study, the authors intend to further increase the number of samples, extend the follow-up time, include as many influencing factors as possible, and divide the patients into IgM deposition group and $\operatorname{IgM}$ negative group, analyze and compare the clinical and pathological features and prognosis of the patients in the two groups to confirm our conclusion.

In summary, we consider that IgM deposition in the mesangial area, Oxford classification, $24 \mathrm{~h}$ urinary protein, pathological type and Lee classification can be used as 
independent influencing factors for poor prognosis of patients with primary IgAN. They will provide evidence for the early detection of high-risk $\operatorname{Ig} \mathrm{AN}$ to establish a reasonable treatment plan, which is of great significance for improving the prognosis of patients with $\operatorname{IgAN}$.

\section{Acknowledgements}

Not applicable.

\section{Funding}

No funding was received.

\section{Availability of data and materials}

The datasets used and/or analyzed during the present study are available from the corresponding author on reasonable request.

\section{Authors' contributions}

YH drafted the manuscript. YH and YZ were mainly devoted to collecting and interpreting the data and $\mathrm{H} \& \mathrm{E}$ staining. $\mathrm{RH}$ and PF were responsible for follow-up. All authors read and approved the final manuscript.

\section{Ethics approval and consent to participate}

The study was approved by the Ethics Committee of West China Hospital of Sichuan University (Chengdu, China). Signed informed consents were obtained from the patients or the guardians.

\section{Patient consent for publication}

Not applicable.

\section{Competing interests}

The authors declare that they have no competing interests.

\section{References}

1. Al Hussain T, Hussein MH, Al Mana H and Akhtar M: Pathophysiology of iga nephropathy. Adv Anat Pathol 24: 56-62, 2017.
2. Daha MR and van Kooten C: Role of complement in $\operatorname{IgA}$ nephropathy. J Nephrol 29: 1-4, 2016.

3. Kusano T, Takano H, Kang D, Nagahama K, Aoki M, Morita M, Kaneko T, Tsuruoka S and Shimizu A: Endothelial cell injury in acute and chronic glomerular lesions in patients with IgA nephropathy. Hum Pathol 49: 135-144, 2016.

4. Yabuki A, Shimokawa Miyama T, Kohyama M and Yamato O: Canine IgA nephropathy: A case report. J Vet Med Sci 78: 513-515, 2016.

5. da Silva LS, Almeida BL, de Melo AK, de Brito DC, Braz AS and Freire EA: IgA nephropathy in systemic lupus erythematosus patients: Case report and literature review. Rev Bras Reumatol Engl Ed 56: 270-273, 2016.

6. Ohata C, Ishii N, Koga H and Nakama T: A clinical and serological study of linear IgA bullous dermatosis without linear immunoglobulin deposition other than $\operatorname{IgA}$ at the basement membrane zone using direct immunofluorescence. Br J Dermatol 177: 152-157, 2017.

7. D'Amico G: Natural history of idiopathic IgA nephropathy and factors predictive of disease outcome. Semin Nephrol 24: 179-196, 2004.

8. Lv J, Hou W, Zhou X, Liu G, Zhou F, Zhao N, Hou P, Zhao M and Zhang $\mathrm{H}$ : Interaction between PLA2R1 and HLA-DQA1 variants associates with anti-PLA2R antibodies and membranous nephropathy. J Am Soc Nephrol 24: 1323-1329, 2013.

9. Patterson R, Schatz M, Fink JN, DeSwarte RS, Roberts M and Cugell D: Pigeons breeders' disease. I. Serum immunoglobulin concentrations; IgG, IgM, IgA and IgE antibodies against pigeon serum. Am J Med 60: 144-151, 1976.

10. Han B, Li Y, Han H, Zhao Y, Pan Q and Ren L: Three IgH isotypes, $\operatorname{IgM}, \operatorname{IgA}$ and $\operatorname{IgY}$ are expressed in Gentoo penguin and zebra finch. PLoS One 12: e0173334, 2017.

11. Lin Q, Chen Y, Lv J, Zhang H, Tang J, Gunaratnam L, Li X andYang L: Kidney injury molecule-1 expression in $\operatorname{IgA}$ nephropathy and its correlation with hypoxia and tubulointerstitial inflammation. Am J Physiol Renal Physiol 306: 885-895, 2014.

12. Sonoda Y, Gohda T, Suzuki Y, Omote K, Ishizaka M, Matsuoka J and Tomino Y: Circulating TNF receptors 1 and 2 are associated with the severity of renal interstitial fibrosis in IgA nephropathy. PLoS One 10: e0122212, 2015.

13. Kosztyu P, Hill M, Jemelkova J, Czernekova L, Kafkova LR, Hruby M, Matousovic K, Vondrak K, Zadrazil J and Sterzl I: Glucocorticoids reduce aberrant O-Glycosylation of IgA1 in IgA nephropathy patients. Kidney Blood Press Res 43: 350-359, 2018.

14. Lloyd IE, Ahmed F, Revelo MP and Khalighi MA: De novo immune complex deposition in kidney allografts: A series of 32 patients. Hum Pathol 71: 109-116, 2018.

15. Wu CK, Yang AH, Lai HC and Lin BS: Combined proximal tubulopathy, crystal-storing histiocytosis, and cast nephropathy in a patient with light chain multiple myeloma. BMC Nephrol 18: 170, 2017.

(i) $\ominus$ This work is licensed under a Creative Commons Attribution-NonCommercial-NoDerivatives 4.0 International (CC BY-NC-ND 4.0) License. 